\title{
PRODUCIR ELECTRICIDAD CON BIOMASA: BENEFICIOS, EXPERIENCIAS Y ACTUALIDAD EN ARGENTINA
}

ADVANTAGES AND CHALLENGES OF BIOMASS POWER GENERATION

\section{BALBINA GRIFFA LEANDRO MARCÓ EVELIN GOLDSTEIN}

Escuela de Economía y Negocios

Universidad Nacional de San Martín ARGENTINA

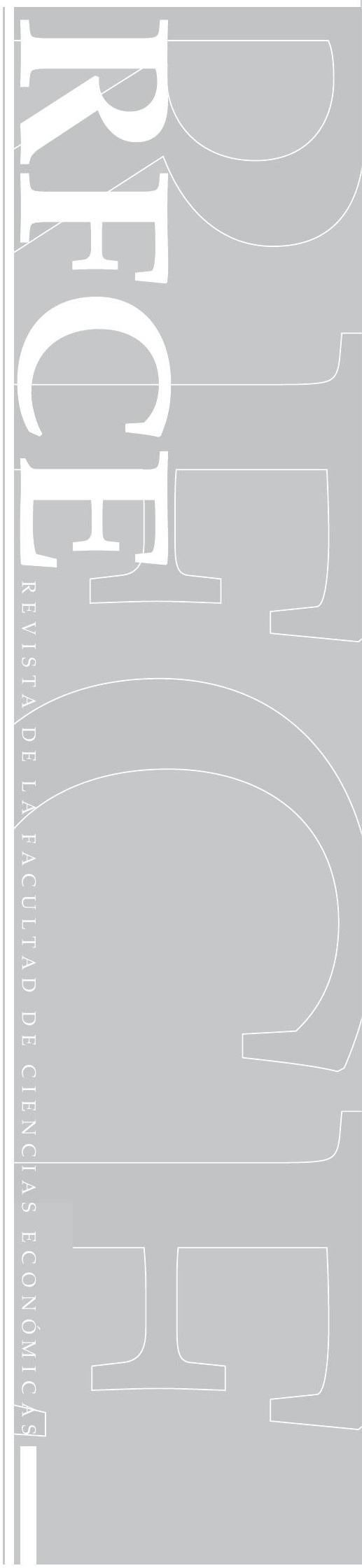




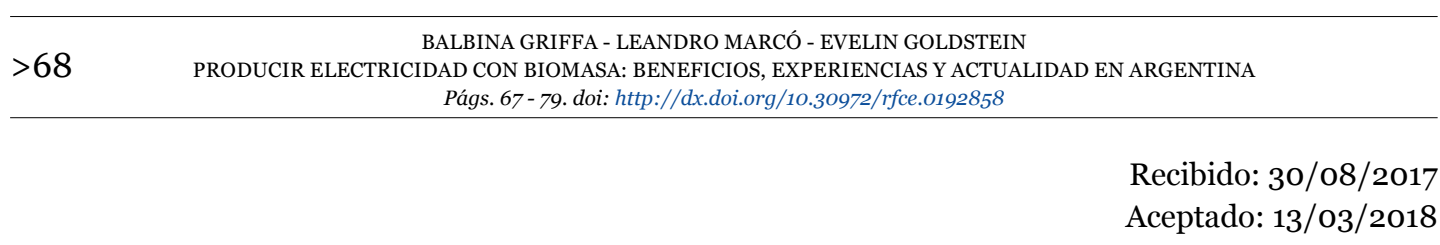

\title{
RESUMEN
}

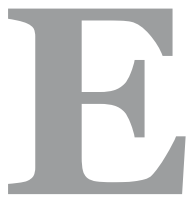

1 nuevo marco normativo para las energías renovables en Argentina, obliga a incrementar la generación eléctrica proveniente de estas fuentes alternativas. La biomasa utilizada como combustible para producir electricidad, es un área poco explorada en nuestro país y con grandes ventajas relativas respecto de otras fuentes renovables. En el presente artículo se estudia la actualidad de la biomasa, sus beneficios, países que tienen una fuerte madurez en el desarrollo y se exhibirán las fortalezas y debilidades encontradas para el desarrollo local de la biomasa.

Palabras clave: biomasa, energías renovables.

\begin{abstract}
The new legal framework for renewable energy in Argentina enforces the development of an area of public interest and commits the government to increasing the share of renewable energies. biomass in Argentina has long been overlooked despite its comparative advantage over other renewable sources. This paper studies the current biomass development, its potential for power generation, as well as some experiences from other countries that have used it. Lastly, this work will exhibit some challenges from the use of biomass in Argentina.
\end{abstract}

Keywords: biomass energy, renewables.

\section{INTRODUCCIÓN}

Las Energías Renovables (ER) son aquellas energías que provienen de recursos naturales que no se agotan y a los que se puede recurrir de manera permanente. Su impacto ambiental es nulo en la emisión de gases de efecto invernadero como el $\mathrm{CO} 2$. Entre ellas se encuentran la energía eólica, la solar fotovoltaica, la geotérmica, la mareomotriz, la pequeña hidráulica y la biomasa.

Las ER se encuentran en pleno desarrollo en el ámbito internacional. El interés por diversificar la matriz energética, el compromiso de reducir las emisiones contaminantes y la aparición de nuevas tecnologías de menor costo son los principales motivos que explican el crecimiento de las inversiones para generar energía a través de fuentes renovables en muchos países del mundo. 
En la Argentina, las ER presentan una penetración aún muy incipiente en comparación con lo que sucede en otros países del mundo. Los recursos energéticos renovables aportan el o,8\% de la energía primaria, mientras que en países como Dinamarca, Finlandia, Alemania y Suecia llegan a dar cuenta de hasta el $20 \%$ del total y en países de la región, como Chile y Brasil, aportan cerca del 5\%. La relevancia de las energías renovables es también pequeña en relación a la demanda de energía eléctrica de nuestro país: solo el 1,9\% del consumo se cubre con generación de fuentes renovables.

El reducido avance de las ER registrado hasta el momento en nuestro país, seguramente no es independiente de la presencia de energéticos convencionales en nuestro territorio. Existe consenso acerca de que la Argentina presenta un gran potencial para el desarrollo de las energías renovables. Entre las diferentes opciones, la energía eólica, la solar y la generación a partir de biomasa aparecerían como las opciones con mayores ventajas naturales.

Sumado a ello, la puesta en vigencia del nuevo marco normativo para las energías renovables, recientemente sancionado, obliga a incrementar la generación eléctrica proveniente de estas fuentes alternativas, al establecer una meta de abastecer el $8 \%$ del consumo de energía eléctrica mediante fuentes renovables al año 2017 (que se fiscalizaría en diciembre de 2018) y el 20\% hacia 2025. Dichas metas deberán ser cumplidas individualmente por los Grandes Usuarios.

Con el nuevo marco normativo el Ministerio de Energía y Minería de la Nación realizó licitaciones para cubrir cupos de energía eléctrica renovable. El resultado de las mismas mostró que existe un fuerte interés de los privados hacia la generación de energía eléctrica eólica y solar.

A partir de ello, en este informe nos interesa estudiar en particular un sector que en nuestro país no se encuentra desarrollado y parecería contar con un gran potencial: la generación de electricidad a partir de biomasa.

\section{DEFINICIÓN DE BIOMASA}

La biomasa es, desde el punto de vista energético, un combustible procedente de productos y residuos naturales (agrícolas o forestales). ${ }^{1}$

Si bien la biomasa, ha sido históricamente utilizada para generar calor en los hogares, en su uso moderno se puede clasificar en tres grandes grupos: biocombustibles líquidos, gaseosos y sólidos.

En el caso de los biocombustibles líquidos, la Argentina ocupa un lugar de importancia a nivel mundial, especialmente en el caso del biodiesel, siendo el tercer productor y primer exportador. Respecto del biocombustible gaseoso, el biogás, en nuestro país es una tecnología explorada aunque en mayor medida son emprendimientos de baja escala. Y, por último, el grupo de los biocombustibles sólidos que es el que estudiamos en este informe, los más importantes son 
los de tipo primario, constituidos por materias lignocelulósicas procedentes del sector agrícola o forestal y de las industrias de transformación que producen residuos de dicha naturaleza.

Desde el punto de vista energético, la biomasa sólida puede utilizarse tanto para usos térmicos como eléctricos. Así pues, la biomasa térmica englobaría aquellas aplicaciones tecnológicas dedicadas al suministro de calor para la calefacción, producción de agua caliente sanitaria (ACS) y/o procesos industriales. Por otro lado, la biomasa eléctrica se centra en aplicaciones para generación de energía eléctrica tanto de forma exclusiva como mediantes sistemas de cogeneración o sistemas de co-combustión. ${ }^{2}$

\section{BENEFICIOS DE PRODUCIR ELECTRICIDAD A PARTIR DE BIOMASA}

El desarrollo de las energías renovables en general permite a los diferentes países que las impulsan resolver al menos dos cuestiones que preocupan a todos los gobiernos:

- Energía local: una de las principales características de la generación eléctrica a partir de ER es que se trata de generación local. El insumo necesario (sol, viento, agua, biomasa, etc.), para la generación de energía eléctrica es local y la generación es propia. Ello presenta algunos beneficios: independencia energética y, en el caso de sustituir importaciones, ahorro de divisas y permite la generación distribuida en base a la potencialidad de cada región (el tipo de tecnología a utilizar se adapta al insumo disponible).

- Reducción de las emisiones de GEI: la posibilidad de reemplazar generación tradicional por ER o bien la posibilidad de ampliar la matriz eléctrica hacia las ER, en cualquiera de los dos casos, se produce una reducción de las emisiones de GEI. Ello ayuda a proteger el medio ambiente y a cumplir con los estándares internacionales, Paris 2015.

En el caso de la biomasa en particular, su uso presenta otras ventajas que nos interesa diferenciar:

- Convertir un residuo en un recurso: se utilizan residuos de otras actividades (principalmente forestal y agrícola) que no tenían un valor económico. En el caso del residuo forestal, según la FAO, se considera que, de cada árbol extraído para la producción maderera, sólo se aprovecha comercialmente un porcentaje cercano al $20 \%$. Se estima que un $40 \%$ es dejado en el campo, en las ramas, despunte y raíces, otro 40\% no aprovechado en la industria de la madera en forma de costaneros, astillas (chips), corteza y aserrín.

- Reducir la disposición de residuos: el uso de la biomasa como fuente energética, constituye un medio para la utilización de grandes cantidades de residuos y con ello una solución a su disposición final. 
- Reducir el riesgo de incendios: tanto en terrenos forestales como agrícolas, la revalorización de los residuos, y por ende su recolección, evita la quema de los mismos en el terreno ya sea intencional o accidentalmente.

- Desarrollo económico de áreas rurales: la planta de generación a partir de biomasa debe construirse en las cercanías donde se encuentra el insumo. La biomasa hay que recolectarla, procesarla, transportarla y maniobrarla. Todas estas actividades le generan un costo adicional a este tipo de generación respecto de otras energías renovables, sin embargo desde una visión más amplia es una ventaja para la zona donde se localizará la planta de generación porque despierta una demanda continua de las actividades vinculadas a hacer llegar la biomasa a la planta y por ende de nuevos puestos de trabajo.

- Balance neutro en emisiones de $\mathrm{CO} 2$ (principal responsable del efecto invernadero). La combustión de biomasa produce $\mathrm{CO} 2$, pero una cantidad análoga a la emitida fue captada previamente por las plantas durante su crecimiento, por lo que la combustión de la biomasa no supone un incremento neto de este gas en la atmósfera3. Es importante señalar que se requiere de una planificación sostenible, con el objetivo de renovar el ciclo de captación del CO2.

- Estabilidad de la oferta: a diferencia de la energía eólica y solar, la energía eléctrica a partir de biomasa es independiente de las condiciones climatológicas.

\section{RECIENTE ACTUALIZACIÓN DEL MARCO NORMATIVO DE ER EN ARGENTINA}

Desde el año 1998, cuando se sancionó la primera Ley4 que declaró de interés la generación a partir de energías renovables hasta la actualidad, nuestro país no ha tenido grandes avances en el área.

En la Ley del año 1998, se fomentaban la generación eólica y solar y para ellos se había fijado como estímulo un sistema de primas por kwh generado, acompañado de ciertos beneficios fiscales. Casi 10 años después, en el 2007, se sanciona una nueva Ley ${ }^{5}$ que amplía el estímulo al resto de las tecnologías capaces de generar energía a partir de fuentes renovables y también incorpora por primera vez una mera participación de las ER en el consumo de energía eléctrica (debía ser el 8\% para el año 2016).

En este contexto, en el año 2009 el Ministerio de Planificación lanzó el GENREN I y II. Este programa consistió en un sistema de licitaciones que pretendía cubrir 1.00o MW de generación con ER. Los resultados no fueron los esperados, menos de 1/3 de los proyectos lograron

\footnotetext{
${ }^{3}$ FEDIT. (2011).

${ }^{4}$ Ley 25.019, crea el Régimen Nacional de Energía Eólica y Energía Solar.

${ }^{5}$ Ley 26.190, Régimen de Fomento Nacional Para el Uso de las Fuentes Renovables de Energía Destinada a la Producción de Energía Eléctrica.
} 
concretarse. En general, los estímulos sancionados no fueron suficientes para superar las barreras económicas y de financiamiento que afronta la puesta en marcha de un proyecto de ER.

Recientemente, en el año 2015, se aprueba la Ley 27.191, donde se declara de interés nacional la generación de energía eléctrica a partir de ER, y se adapta y mejora el marco regulatorio con el objetivo de incrementar su participación y diversificar la matriz energética nacional.

La nueva ley impulsa el compromiso para los Grandes Usuarios de energía eléctrica (aquéllos con una demanda de potencia mayor o igual que $300 \mathrm{~kW}$ ) de alcanzar un porcentaje de participación del $8 \%$ de renovables para fin de $2017^{6}$ en el consumo total de electricidad, imponiendo una multa para aquellos usuarios que no cumplan ${ }^{7}$. Buscando brindar una solución a las barreras de financiamiento del sector, la nueva Ley crea el Fondo para el Desarrollo de las ER (FODER), que tiene como objetivo el otorgamiento de préstamos, realizar aportes de capital en sociedades, bonificar puntos porcentuales de la tasa de interés financiera, otorgar avales y garantías para respaldar los contratos de compra venta de energía.

En cuanto a la biomasa en particular, a finales de 2012 el Ministerio de Agricultura, Ganadería y Pesca y el Ministerio de Planificación Federal, Inversión Pública y Servicios, con la asistencia técnica de la FAO lanzan el proyecto para la promoción de la energía derivada de biomasa (PROBIOMASA). El objetivo de este proyecto era generar la información, capacitación, asesoramiento, entre otros, en proyectos de producción de energía térmica o eléctrica a partir de biomasa y acercar a los potenciales beneficiarios las opciones de asistencia financiera ya existentes para proyectos que pudieran ser elegibles.

\section{ESTUDIO DE EXPERIENCIAS: ALEMANIA Y FINLANDIA}

Numerosos países han desarrollado políticas e incentivos para estimular las fuentes de energía renovables. La preocupación por el cambio climático, la seguridad energética y acceso de grupos vulnerables a la energía, han generado una amplia gama de instrumentos de política económica tendientes a fomentar el desarrollo de las energías limpias. En general, estos ilustran su alto costo relativo en comparación con las fuentes tradicionales de generación energética, los respectivos límites de financiamiento desde el sector privado y las destrezas y enfoques especiales que generan.

La mayoría de los gobiernos han establecido metas de participación de las fuentes renovables en el consumo de energía eléctrica para los próximos años y, en algunos casos, se combinan con objetivos de reducción de emisiones y de eficiencia energética. Los mecanismos para motivar el crecimiento de las energías renovables, no obstante, difieren entre países, encontrándose, por

\footnotetext{
${ }^{6}$ Se fiscalizaría en diciembre de 2018.

${ }^{7}$ Los usuarios que no cumplan deberán abonar el faltante a un precio igual al Costo Variable de Producción de Energía Eléctrica correspondiente a la generación de gasoil de origen importado.
} 
ejemplo, políticas de subsidio de tarifas para la generación renovable, impuestos sobre el uso de combustibles fósiles o sobre las emisiones producidas, incentivos para inversiones, apoyo a la investigación e innovación científica sectorial, entre otros. Aunque es posible encontrar que ha sido común que las estrategias iniciales de fomento hayan contado con una fuerte presencia estatal.

A continuación se presentan dos países que presentan grandes avances en lo que refiere a la producción de electricidad y calor a partir de biomasa. Ellos son Alemania y Finlandia.

\section{ALEMANIA}

En Alemania se inició en 1991 con la política de fomento a las energías renovables, esencialmente a partir de pagos compensatorios para los proyectos de generación de energía, los cuales se otorgaban por un período de 20 años. Luego, en el año 2000 se sancionó la ley de Energía Renovable (EEG ${ }^{8}$ ), para contar con un marco normativo más completo, la cual ha sido actualizada sucesivamente hasta la actualidad.

En la EEG se determinan, entre otros, las metas globales de sustitución de consumo eléctrico convencional por renovable, en el año 2014 se actualizaron los valores a: 40-45\% del consumo bruto eléctrico en 2025, 55/60\% al 2035 y un mínimo de 80\% al 2050. La ley también incluye metas de eficiencia energética, esperando una reducción en el consumo total de electricidad en un $10 \%$ en 2020 y $25 \%$ en 2050 . En la normativa se establece también la prioridad de acceso a la red para la producción de energía eléctrica solar, eólica y de biomasa, lo que resulta clave para motivar el establecimiento de plantas de generación renovable.

Después de 25 años de vigencia, Alemania reformó el esquema de incentivos para las energías renovables, con la eliminación del feed-in tariff, por el actual sistema de subastas para asignar potencia de generación eléctrica por tecnologías.

En el caso de la biomasa en particular, en el 2016 se incluyó dentro del sistema de subastas de energía y se establecieron metas de incorporar $150 \mathrm{MW}$ anuales en los próximos tres años y $200 \mathrm{MW}$ en los siguientes tres.

En el desarrollo de la Biomasa, uno de los obstáculos con los que se encontró este país fue un fuerte incremento en el uso de la tierra para cultivos energéticos, a partir de lo cual se estableció un límite máximo para destinar tierras con este fin y, al mismo tiempo, introdujeron nuevos incentivos para el uso de residuos de la biomasa.

En Alemania, a partir del año 2008 cuentan con centro de investigación de biomasa, (German Biomass Research Center) ${ }^{9}$ donde se focalizan los principales esfuerzos de innova-

${ }^{8}$ Erneuerbare-Energien- Gesetz, su nombre original en Alemán.

${ }^{9} \mathrm{DBFZ}$, por sus siglas en alemán. 
ción tecnológica para el uso de la biomasa como fuente de energía. Una de las líneas de investigación se sustenta en consolidar lo que se denomina "cluster de bioeconomia", utilizar la disponibilidad de biomasa, transformándola en insumos para diversas cadenas de valor, entre ellos la generación de energía eléctrica.

\section{FINLANDIA}

Finlandia se inicia con la promoción de la bioenergía con la aplicación de impuestos sobre los combustibles fósiles a principios de la década del 90'. Sin embargo, el primer plan integral para fomentar las ER fue publicado en el año 1999, y en el año 2005 se aprobó la Estrategia Nacional Energética y Climática (National Energy and Climate Strategy), la cual fue actualizada en 2008 y 2013 (IEA, 2013). En dicha Estrategia se fijó la meta de sustitución de energía fósil por renovable para alcanzar una participación del 38\% para el año 2020 (en el 2014 ya se cumplía la meta).

Los objetivos de generación eléctrica de fuentes renovables se imponen por tecnología, siendo la biomasa la de mayor contribución esperada (103 TWh de consumo energético final para el año 2020). También se establecieron objetivos de eficiencia energética, que consistieron en que en el año 2020 el consumo energético total no debería superar los 310 TWh (lo que implica una reducción de 13 TWh con respecto al consumo de 2010).

La generación de electricidad en base a fuentes de energía renovable se promueve en Finlandia principalmente a través de sistemas feed-in tariffs. Las fuentes de energía renovable alcanzadas por este mecanismo son la eólica, la biomasa y el biogás. Los contratos de largo plazo se celebran por un máximo de 12 años con precios subsidiados cuyo monto es la diferencia entre el precio objetivo y el promedio del precio de mercado de los anteriores tres meses, con un tope máximo. El uso de las fuentes renovables para la calefacción se fomenta principalmente a través del bonus adicional que se establece para plantas de esquemas combinados que usan biogás y madera.

En Finlandia, por su parte, se le asigna un papel muy importante a la bioeconomía. La abundancia de recursos boscosos y el alto nivel de experiencia en el manejo de la cadena forestal y las fortalezas del sector industrial ubican al país como pionero en bioeconomía a nivel mundial. Allí, es frecuente la utilización de biomasa forestal para la producción de energía a nivel industrial y municipal con plantas de generación combinada de calor y electricidad (CHP), alcanzando una mayor eficiencia energética. Las calderas son generalmente para diferentes tipos de combustibles.

El sector de fabricación de maquinaria también se ha focalizado en la producción de equipos para la cadena foresto-industrial. En igual sentido, existe un énfasis en la orientación de recursos para investigación buscando incorporar prácticas más avanzadas en la conversión de biomasa en energía. 
Cabe señalar que al igual que en Alemania, en Finlandia se busca fomentar el establecimiento de terminales multipropósito, especialmente desde centros de investigación aplicada ${ }^{10}$. El objetivo es incrementar el uso sostenible de la materia prima hacia diferentes productos, sin generación de desperdicios, y para la agregación de valor. El proceso implica la recepción de la biomasa forestal (y otros materiales) en la terminal, donde se procesa, limpia, clasifica y separa. A partir de ello, se utilizará para combustible para generación combinada pero también para fabricación de productos cosméticos, alimenticios, para la industria química, textil, papel y empaques.

\section{FORTALEZAS Y DEBILIDADES PARA EL DESARROLLO DE ENERGÍA A PARTIR DE BIOMASA EN ARGENTINA}

Argentina, posee una vasta agricultura además de recursos forestales. Según un informe de la FAO, nuestro país cuenta con gran cantidad de recursos biomásicos que no se utilizan. En particular, la FAO cuantifica aquellos correspondientes a la forestoindustria, identificando a las provincias de Misiones, Corrientes, Entre Ríos y Buenos Aires.

A partir del estudio de este sector en particular, nos proponemos a continuación realizar un análisis de las fortalezas y debilidades que encuentra el desarrollo de la generación eléctrica a partir de biomasa en las provincias que cuentan con el recurso.

\section{Fortalezas:}

- Según la FAO, la Argentina posee una cantidad de recursos biomásicos forestales importantes, de los cuales solo una muy pequeña parte es utilizada en la actualidad, existiendo en consecuencia disponibilidad de recursos para futuros aprovechamientos.

- Nuestro país se caracteriza por su despliegue de agricultura, o con extensiones de tierras como para poder realizar cultivos energéticos.

- Las provincias que cuentan con actividad agrícola también, podrían seguir el caso de Finlandia, invirtiendo en centrales que generen calor (para el secado de granos) y electricidad, ello hace un rendimiento más eficiente.

- El desarrollo de dicha actividad podría ser la fuente de generación de valor agregado y empleo en zonas rurales.

- Al contar con el recurso, nuestro país podría, al igual que Finlandia, planificar y desarrollar tecnología. Dirigir recursos económicos a la I\&D de la cadena de valor de la biomasa.

- Podría ser una solución económica para las industrias que generan estos tipos de desperdicios para cumplir con el $8 \%$ de consumo de EE a partir de ER.

- Nuestro país cuenta con la experiencia de los biocombustibles, donde logró desarrollar una industria competitiva.

${ }^{10}$ Universidad JAMK e instituto VTT. 
- Posibilidad de instrumentar terminales multipropósito como en Finlandia, con el objetivo de centralizar los "residuos" biomasa por áreas y explotar al máximo su reutilización.

\section{Debilidades:}

- En principio el impulso de las ER en nuestro país es nacional, y estos proyectos tienen una importancia mucho mayor para las ciudades donde se realiza que para el país. Son proyectos de baja escala para la perspectiva nacional, pero de alto impacto a nivel local.

- No aparecen algunos estímulos que fueron claves en el desarrollo de la generación a partir de biomasa en algunos casos estudiados: los incendios forestales no son muy frecuentes en nuestro país. Así como, la explotación de la biomasa para generar calor, en principio, en nuestro país compite directamente con el gas natural que es un combustible más económico.

- Costo de generación más elevados en relación a otras tecnologías. El sector presenta externalidades positivas, que de no ser consideradas dificulta la competitividad frente a otros tipos de proyectos. Es necesario realizar estudios que valoricen las externalidades positivas generadas por esta actividad.

- La FAO identifica como barreras técnicas la insuficiente información sobre los recursos y falta de infraestructura donde se localizan los recursos.

- Falta de estímulos específicos al sector en particular.

- Según un estudio de la FAO, si bien existe tecnología apropiada para este tipo de emprendimientos, es limitada en nuestro país. No existen fabricantes de todos los componentes de una central, y es de interés inducir a que empresas locales construyan motores a vapor para bajar costos con la finalidad de su inserción en el medio productivo.

- Las dificultades de conseguir financiamiento de largo plazo para este tipo de proyectos. Por ello es crucial el interés del gobierno local por impulsar esta actividad.

\section{ACTUALIDAD DE LA GENERACIÓN A PARTIR DE BIOMASA}

Si bien existen algunos proyectos de índole privado que se encuentran funcionando, según datos del programa PROBIOMASA, las jurisdicciones provinciales que registran un mayor desarrollo e interés en el rubro son Buenos Aires, Córdoba, Entre Ríos y Santa Fe. En este relevamiento se incluyen proyectos de biogás también.

En un segundo grupo de interés, aparecen Jujuy, Misiones, Chubut, Salta, San Juan, Tucumán y Tierra del Fuego.

Los resultados de la convocatoria a la presentación de proyectos de generación de energía eléctrica a partir de fuentes renovables "Programa Renovar - Ronda 1" (octubre 2016) alcan- 
zaron un total de 1.143 MW de nueva potencia, a través de 29 proyectos (eólica, solar, biogás, biomasa y pequeños aprovechamientos hidráulicos). El precio promedio de todos los proyectos fue de usd 63 por $\mathrm{Mw} / \mathrm{h}$.

\begin{tabular}{|l|c|c|c|c|c|}
\hline \multicolumn{7}{|c|}{ Resultados Renovar 1 - Biomasa } \\
\hline $\begin{array}{c}\text { Nombre del } \\
\text { proyecto }\end{array}$ & $\begin{array}{c}\text { Potencia en } \\
\text { MW }\end{array}$ & Oferente & Provincia & Localidad & $\begin{array}{c}\text { Precio en } \\
\text { usd }\end{array}$ \\
\hline $\begin{array}{l}\text { Generación Biomasa } \\
\text { Santa Rosa }\end{array}$ & 13 & $\begin{array}{c}\text { Genergiabio } \\
\text { Corrientes S.A. }\end{array}$ & Corrientes & Santa Rosa & 110 \\
\hline Pindó Eco & 2 & Pinedo S.A. & Misiones & $\begin{array}{c}\text { Puerto } \\
\text { Esperanza }\end{array}$ & 110 \\
\hline
\end{tabular}

Fuente: http://portalweb.cammesa.com/pages/renovar.aspx

En el caso de particular de la biomasa, se presentaron 2 proyectos por un total de $15 \mathrm{MW}$ de potencia a un precio promedio de usd 110 por $\mathrm{Mw} / \mathrm{h}$. Así esta tecnología representó el 1,3\% del total de la licitación y el precio un 75\% mayor al precio promedio por Mw/h de la licitación.

\section{CONSIDERACIONES FINALES}

No es casual que un país como la Argentina, que cuenta con recursos no convencionales (petróleo + gas natural) haya demorado un poco más en desarrollar las energías renovables respecto a cualquier otro país que no cuente con hidrocarburos. La ventaja de la independencia energética que otorga a muchos países el desarrollo de los renovables, no es tan significativa para aquellos que si cuentan con recursos convencionales.

La historia reciente de nuestro país muestra que los programas de estímulo a las ER hasta la actualidad no han logrado el desarrollo a escala de las mismas. La nueva ley aprobada y reglamentada recientemente abre un nuevo escenario, al contar con nuevas reglamentaciones. Sin embargo, para concretar los proyectos será clave la intervención del Estado como garante de la obtención de los recursos (financiación) necesarios para realizar estas inversiones de largo plazo.

El impulso a la generación de energías renovables, y en particular a partir de biomasa, es una oportunidad para nuestro país que cuenta con el recurso, además de contribuir al cuidado del medio ambiente y cumplir con estándares internacionales, contribuyen a generar un mayor nivel de actividad económica y nuevos puestos de trabajo.

Es importante revisar la experiencia internacional que nos muestra cómo avanzaron países que ya tienen desarrollado este sector, los obstáculos con los que se encontraron y el camino exitoso hacia mercados más grandes como una nueva área de innovación tecnológica o el desarrollo de la bioeconomía. 
De acuerdo a lo expuesto, en la Argentina las condiciones para la generación de energía eléctrica a partir de la biomasa están dadas, el éxito dependerá de las acciones que se encaren para resolver los problemas existentes, que desde luego está en gran medida en manos del sector privado.

\section{REFERENCIAS BIBLIOGRÁFICAS}

Cámara Argentina de Energías Renovables. (2015). Aportes para un sistema eléctrico eficiente y sustentable. Desarrollo industrial y de las economías regionales. Reporte Ejecutivo 2015. Recuperado de www.cader.org.ar

DBFZ. (2015). Annual Report 2015. Recuperado de http://www.dbfz.de

Elija A.. (2016). Biomass policies. National Policy landscapes: Finland. VTT Institute. Recuperado de http://biomassproducer.com.au/starting-a-bioenergy-project/policies-andregulations/\#.WwMoaO4vyM8

European Commission. (2014). Finland's National Energy Efficiency Action Plan. NEEAP-3, Report pursuant to Article 24(2) of the Energy Efficiency Directive (2012/27/EU) to the European Commission, 1094/832/2014. Recuperado de http://www.ec.europa.eu

FEDIT. (2011). "Biomasa. Oportunidades para el sector de fabricantes de Bienes de Equipo". Observatorio Industrial del Sector de Fabricantes de Bienes de Equipo. Recuperado de http://www.minetad.gob.es

International Energy Agency. (2013). Energy policies of IEA countries, Finland, 2013 Review. Recuperado de http://www.oecd-ilibrary.org

Morris G.. (2000). Biomass Energy Production in California: The Case for a Biomass Policy Initiative. Final Report. Recuperado de https://www.nrel.gov/

Morrone M. (2016). Energías Renovables en Argentina Programa RenovAr - Ronda 1 -Primera Convocatoria Abierta por 1000 MW. Dirección Nacional de Promoción de Energías Renovables. Recuperado de http://www.probiomasa.gob.ar 
Rojas A. \& Tubio B.. (2015). La retribución de las energías renovables: retos e incertidumbres. Recuperado de http://www.afi.es

Uasuf A. \& Hilbert J.. (2012). El uso de la biomasa de Origen Forestal con destino a bioenergía en la Argentina. Informes Técnicos Bioenergía, Año $1 \mathrm{~N}^{\circ} 3$. Recuperado de http://www.probiomasa.gob.ar

Sitios consultados:

www.cammesa.com.ar

www.infoleg.gob.ar

www.calbiomass.org

www.energy.ca.gov

www.ren21.net

www.eia.gov

\section{CURRICULUM VITAE}

\section{Balbina Griffa}

Lic. en Economía (UBA). Especialista en Mercado Eléctrico y Gas Natural (ITBA). Directora del Centro de Investigación en Economía y Planeamiento Energético de la EEyN de la UNSAM. Docente EEyN, UNSAM.

bgriffa@unsam.edu.ar

\section{Evelin Goldstein}

Lic. en Economía (UBA). Mgter. en Políticas Públicas (UTDT). Docente de Economía de la Producción en la Facultad de Agronomía (FAUBA).

egolds81@gmail.com

\section{Leandro Marcó}

Licenciado en Economía (UADE), elaborando la tesis de la Maestría Interdisciplinaria en Energía (UBA). Docente EEyN-UNSAM y FCE-UBA. Investigador en el Centro de Investigación en Economía y Planeamiento Energético (EEyN de la UNSAM).

lmarco@unsam.edu.ar 